\title{
ON MOBILITY AND EINSTEIN RELATION FOR TRACERS IN TIME-MIXING RANDOM ENVIRONMENTS.
}

\author{
TOMASZ KOMOROWSKI AND STEFANO OLLA
}

\begin{abstract}
In this paper we rigorously establish the existence of the mobility coefficient for a tagged particle in a simple symmetric exclusion process with adsorption/desorption of particles, in a presence of an external force field interacting with the particle. The proof is obtained using a perturbative argument. In addition, we show that, for a constant external field, the mobility of a particle equals to the self-diffusivity coefficient of the medium, the so-called Einstein relation. The method can be applied to any system where the environment has a Markovian evolution with a fast convergence to equilibrium (spectral gap property). In this context we find a necessary relation between forward and backward velocity for the validity of the Einstein relation. This relation is always satisfied by reversible systems. We provide an example of a non-reversible system, where the Einstein relation is correct.
\end{abstract}

\section{INTRODUCTION}

In his celebrated paper on a Brownian motion (cf. [5]) Einstein established a linear relation between the mobility $\sigma$ and the diffusivity $D$ of a Brownian particle. The diffusivity of a Brownian particle is defined as

$$
D t=\mathbb{E}|\mathbf{X}(t)|^{2} .
$$

The mobility $\sigma$ is defined in the following way. Suppose that an exterior uniform force field $\mathbf{E}$ is applied to the particle, then, in the corresponding stationary state, the particle will have a velocity $\mathbf{v}(\mathbf{E})$ corresponding to the value $\mathbf{E}$ of the field. The limit

$$
\lim _{|\mathbf{E}| \rightarrow 0+} \frac{|\mathbf{v}(\mathbf{E})|}{|\mathbf{E}|}=\sigma
$$

defines the mobility. The Einstein relation says that $\sigma=\beta D$, where $\beta^{-1}=k_{B} T, T$ being the temperature of the environment fluid, and $k_{B}$ is the Boltzmann constant. A heuristic derivation of this relation can be found in section 8.8 of [16].

A rigorous derivation of Einstein relation from a purely mechanical system is quite a difficult open problem. It is natural to look first at those models where the convergence to Brownian motion (of the rescaled path) is known, like for certain tracer dynamics in

Date: September 6, 2004.

ACKNowledgements. We wish to express our thanks to the anonymous referee whose comments lead to a significant improvement of the manuscript. In particular we benefited from the remarks of the referee while preparing Example 4.3. Also, the section 4 was inspired by a suggestion made by the referee. The research of the first author was supported by KBN grant nr 2PO3A 03123 and by the grant from the European Science Foundation. 
stochastic environments (cf. references at the end of chapter 8 in [16]). For a different approach concerning weak perturbation of the dynamics see [10].

The main difficulty one finds when trying to prove an Einstein relation, lies in establishing the existence of a stationary state and have good properties of relaxation to this stationary state of the dynamics (cf. [3] for a negative result on a deterministic Hamiltonian dynamics of interacting particles).

In a recent paper (cf. [13]) M. Loulakis considered this problem for a tagged particle in the symmetric simple exclusion in dimension 3 or higher. In this model the environment dynamics, even though stochastic, slowly converges to an equilibrium, because of the conservation law of the number of particles. Remarkably Loulakis, without proving the existence of a unique stationary state nor the existence of the effective velocity, was able to prove a form of the Einstein relation.

In this paper we consider systems whose environment dynamics have very good properties of relaxation to equilibrium (essentially they are Markovian with a unique invariant measure, and with a gap in the spectrum of the generator). A simple example of a system of this type is provided by the symmetric simple exclusion with creation and destruction of particles. A perturbative argument permits then to prove, see theorem 2.2, for perturbations not too big with respect to the gap of the spectrum of the generator, the existence of a stationary state that is absolutely continuous with respect to the equilibrium state. In addition we obtain exponentially fast in time relaxation to this stationary state, i.e. the stationary state is stable in the sense of (2.19). The method allows in principle to compute all terms of the perturbative expansion of the effective velocity.

Once these properties of the stationary state of the perturbed system are established, one can study the mobility for a tracer particle in the following fashion. For simplicity we restrict ourselves, to the one-dimensional case (the detailed argument is exposed in section 4.3). In the unperturbed system, the position $x_{t}$ at time $t$ of the tracer is given by an additive functional of $\left\{\xi_{s}\right\}_{0 \leq s \leq t}$, the (unperturbed) environment dynamic as seen from the tracer. Typically this functional is antisymmetric with respect to time inversion (cf. [4]) and can be written in the standard decomposition form:

$$
x_{t}=\int_{0}^{t} \psi\left(\xi_{s}\right) d s+M_{t},
$$

where $M_{t}$ is a square integrable martingale with respect to the filtration generated by $\left\{\xi_{t}\right\}_{t \geq 0}$.

We denote by $\mu$ the stationary measure for the unperturbed environment dynamics. The function $\psi$ appearing in (1.3) is usually called the mean forward velocity and we assume it belongs to $L^{2}(\mu)$ and has null $\mu$ average. It has the property

$$
\lim _{t \rightarrow 0} \frac{1}{t} \mathbb{E}_{\mu}\left(x_{t} f\left(\xi_{0}\right)\right)=\int \psi f d \mu
$$

for any $f \in L^{2}(\mu)$.

The mean backward velocity is defined by

$$
\lim _{t \rightarrow 0} \frac{1}{t} \mathbb{E}_{\mu}\left(x_{t} f\left(\xi_{t}\right)\right)=-\int \psi^{*} f d \mu
$$


We assume that $\psi^{*}$ exists in $L^{2}(\mu)$ and has null $\mu$ average. Observe that if $\mu$ is reversible, by the antisymmetry of $x_{t}$ for time reversal, we have $\psi=\psi^{*}$.

The asymptotic variance of the tracer is then given by

$$
D=\lim _{t \rightarrow \infty} \frac{1}{t} \mathbb{E}_{\mu}\left(x_{t}^{2}\right)=\mathbb{E}_{\mu}\left(M_{1}^{2}\right)-2 \int_{0}^{\infty} \mathbb{E}_{\mu}\left[\psi^{*}\left(\xi_{0}\right) \psi\left(\xi_{t}\right)\right] d t .
$$

The assumptions made about the dynamics are more than sufficient to establish the existence of this limit and the convergence of the integral on the right hand side of (1.6).

One can now perturb the dynamics by applying a uniform external field of strength $\alpha$ on the tracer. This can be done on the level of the path measure by a Girsanov transformation (in the spirit of [10]). Because of the good properties of the stationary state $\mu_{\alpha}$ of the perturbed dynamics, there will exists an asymptotic velocity for the position of the tracer defined as

$$
\lim _{t \rightarrow \infty} \frac{x_{t}^{\alpha}}{t}=v(\alpha)
$$

and it turns out that the mobility is given by

$$
\sigma=: \lim _{\alpha \rightarrow 0} \frac{v(\alpha)}{\alpha}=D+\int_{0}^{\infty} \mathbb{E}_{\mu}\left\{\left[\psi^{*}\left(\xi_{0}\right)-\psi\left(\xi_{0}\right)\right] \psi\left(\xi_{t}\right)\right\} d t
$$

so the Einstein relation is valid if and only if the second term on the right hand side of (1.7) is null.

In reversible systems (i.e. if $\mu$ satisfies a detailed balance condition) we always have $\psi=\psi^{*}$. In the example 4.3 below we show a non-reversible system where $\psi=\psi^{*}$.

We have chosen to expose the method first in the concrete example of the symmetric simple exclusion with creation and annihilation (sections 2 and 3), while the general case and some more examples are treated in section 4.

The one dimensional nearest-neighbor case for the exclusion with creation-annihilation was treated in [1], where a mean field approximate solution is proposed.

\section{SyMmetric Simple EXCLUSION PROCESS With ADSORPtiOn/DESORPtion.}

This process can be informally described as follows. Suppose that $p(\cdot)$ is a finite range probability measure on $\mathbb{Z}^{d}$ such that $p(-x)=p(x)$. Let $K$ be such that $p(x) \equiv 0$ for $|x| \geq K$, and $S_{p}:=\left[x \in \mathbb{Z}^{d}: p(x)>0\right]$ and $p_{0}:=\inf \left[p(x): x \in S_{p}\right]>0$. We assume that $S_{p}$ generates all $\mathbb{Z}^{d}$. Consider now an initial configuration of particles. A particle in the site $x$, independently of the other particles, waits for an exponential time of intensity $p(x-y)$ and attempts a jump to the site $y$. If the site $y$ is already occupied by another particle the jump is suppressed. Also each particle can disappear with intensity $\rho \in[0,1]$ and in each empty site can be created with intensity $1-\rho$.

A natural state space for the process is $\Omega=\{0,1\}^{\mathbb{Z}^{d}}$. It consists of all possible configurations $\eta \in \Omega, \eta(x)=0$, or 1 depending on whether the site $x \in \mathbb{Z}^{d}$ is occupied, or not.

One can show, see Theorem 3.9 p. 27 of [11], that such a stochastic process $\eta_{t}, t \geq 0$ can be well defined from each initial configuration $\eta$. A standard procedure consists in constructing the $C_{0}$-semigroup $\left(P^{t}\right)_{t \geq 0}$ on $C(\Omega)$, the space of continuous functions on $\Omega$. The class $\mathcal{C}$ of 
local functions, i.e. functions depending on finitely many sites, is a core of the generator $L$ of $\left(P^{t}\right)_{t \geq 0}$ and

$$
\begin{array}{r}
L f(\eta)=L_{\text {jump }} f(\xi)+L_{a d} f(\xi)=\sum_{x, y} p(y-x) \eta(x)(1-\eta(y))\left(f\left(\eta^{x, y}\right)-f(\eta)\right) \\
+\sum_{x}[\rho(1-\eta(x))+(1-\rho) \eta(x)]\left(f\left(\eta^{x}\right)-f(\eta)\right) .
\end{array}
$$

Here

$$
\eta^{x, y}(z)= \begin{cases}\eta(z), & \text { if } z \notin\{x, y\} \\ \eta(y), & \text { if } z=x \\ \eta(x), & \text { if } z=y\end{cases}
$$

The configuration $\eta^{x}$ is defined by

$$
\eta^{x}(z)= \begin{cases}\eta(z), & \text { if } z \neq x \\ 1-\eta(x), & \text { if } z=x\end{cases}
$$

A simple calculation shows that the product measure $\nu_{\rho}:=\bigotimes_{\mathbb{Z}^{d}} B(\rho)$, where $B(\rho)$ is the Bernoulli measure with success probability $\rho$, is a unique invariant and ergodic measure for the process. Since the generator $L$ is symmetric with respect to $\nu_{\rho}$, this measure is reversible. The semigroup $\left(P^{t}\right)_{t \geq 0}$ extends to a semigroup of self-adjoint operators on $L^{2}\left(\nu_{\rho}\right)$, which with some abuse of notation we denote by the same symbol. This semigroup possesses a spectral gap $\lambda_{0}>0$, i.e.

$$
\left\|P^{t} f\right\|_{L^{2}\left(\nu_{\rho}\right)} \leq e^{-\lambda_{0} t}\|f\|_{L^{2}\left(\nu_{\rho}\right)} \text { for all } f \text {, s.t. } \int f d \nu_{\rho}=0 .
$$

One can easily see that $\lambda_{0} \geq 1$. In fact, 1 is the spectral gap for the creation and annihilation part $L_{a d}$ of the generator, and the jump part can only increase this gap.

2.1. Tagged particle process. We start now with an initial configuration where there is a special particle in the position 0 and we tag it. Let us denote its position at time $t$ by $x_{t}$. Furthermore, we assume that the tagged particle cannot be absorbed.

The environment process $\left(\xi_{t}\right)_{t \geq 0}$ is defined on a slightly modified state space $\Omega:=\{0,1\}^{\mathbb{Z}_{*}^{d}}$, where $\mathbb{Z}_{*}^{d}:=\mathbb{Z}^{d} \backslash\{0\}$, via $\xi_{t}(x):=\eta_{t}\left(x+x_{t}\right)$. It describes the environment "as seen from the tagged particle". The generator $\mathcal{L}_{0}$ of the process can be written on local functions as follows, cf. pp. 278-279 of [12]

$$
\begin{array}{r}
\mathcal{L}_{0} f(\xi)=L_{\text {jump }} f(\xi)+L_{a d} f(\xi)+\mathcal{L}_{\tau} f(\xi)=\frac{1}{2} \sum_{x, y \neq 0} p(y-x)\left(f\left(\xi^{x, y}\right)-f(\xi)\right) \\
+\sum_{x \neq 0}[\rho(1-\xi(x))+(1-\rho) \xi(x)]\left(f\left(\xi^{x}\right)-f(\xi)\right)+\sum_{x \neq 0} p(x)(1-\xi(x))\left(f\left(\tau_{x} \xi\right)-f(\xi)\right)
\end{array}
$$

Here we have defined

The following result holds.

$$
\tau_{x} \xi(z)= \begin{cases}\xi(z+x), & \text { if } z \neq-x \\ \xi(x), & \text { if } z=-x\end{cases}
$$


Proposition 2.1. $\mu(\cdot):=\nu_{\rho}(\cdot \mid \xi(0)=1)$ is an ergodic, invariant and reversible measure for the environment process $\left(\xi_{t}\right)_{t \geq 0}$. Its transition of probability semigroup $\left(Q^{t}\right)_{t \geq 0}$ extends to a $C_{0}$-semigroup of self-adjoint operators on $L^{2}(\mu)$. The Dirichlet form associated with this process is given by

$$
\mathcal{D}(f):=-\left(\mathcal{L}_{0} f, f\right)_{L^{2}(\mu)}=\mathcal{D}_{0}(f)+\mathcal{D}_{a d}(f)+\mathcal{D}_{\tau}(f),
$$

and

$$
\begin{gathered}
\mathcal{D}_{0}(f):=-\left(L_{0} f, f\right)_{L^{2}(\mu)}=\frac{1}{4} \sum_{x, y \neq 0} p(y-x) \int\left[f\left(\xi^{x, y}\right)-f(\xi)\right]^{2} \mu(d \xi) \\
\mathcal{D}_{a d}(f)=\rho(1-\rho) \sum_{x \neq 0} \int\left[f\left(\xi^{x}\right)-f(\xi)\right]^{2} \mu(d \xi) \\
\mathcal{D}_{\tau}(f):=-\left(\mathcal{L}_{\tau} f, f\right)_{L^{2}(\mu)}=\frac{1}{2} \sum_{x \neq 0} p(x) \int(1-\xi(x))\left[f\left(\tau_{x} \xi\right)-f(\xi)\right]^{2} \mu(d \xi), \quad f \in \mathcal{C} .
\end{gathered}
$$

It satisfies the spectral gap estimate

$$
\mathcal{D}(f) \geq \lambda_{0}\|f\|_{L^{2}(\mu)}^{2}, \quad \forall f \in \mathcal{C} \cap L_{0}^{2}(\mu),
$$

where $\lambda_{0}$ is the same as in (2.4) and $L_{0}^{2}(\mu):=\left[f \in L^{2}(\mu) \int f d \mu=0\right]$.

Proof. The proof of the above proposition is standard. The calculation that $\mu$ is an invariant and reversible measure is essentially the same as the one presented in Lemma 2.1 of of [8]. The spectral gap estimate for $L_{a d}$ part of the generator, cf. (2.1), implies the spectral gap estimate (2.9), which in turn implies ergodicity of $\mu$. The formulas for the quadratic forms $\mathcal{D}_{0}(\cdot), \mathcal{D}_{a d}(\cdot), \mathcal{D}_{\tau}(\cdot)$ are obtained by a direct calculation.

The 1-direction coordinate of the position of the particle can be represented by a jump process

$$
x_{t} \cdot \mathbf{l}=\sum_{s \leq t} \sum_{z} z \cdot \mathbf{l} \chi_{\left[\tau_{z} \xi_{s}=\xi_{s-}\right]}=\int_{0}^{t} \psi\left(\xi_{s}\right) \cdot \mathbf{l} d s+M_{t}^{\mathbf{l}} \text {, }
$$

where

$$
\psi(\xi)=\sum_{z \in \mathbb{Z}^{d}} z p(z)(1-\xi(z))
$$

and $\left(M_{t}^{1}\right)_{t \geq 0}$ is a cadlag martingale w.r.t. the natural filtration of $\xi_{t}$ whose quadratic variation equals

$$
\left\langle M^{\mathbf{l}}\right\rangle_{t}=\int_{0}^{t} \sum_{z}(z \cdot \mathbf{l})^{2} p(z)\left(1-\xi_{s}(z)\right) d s .
$$

Since

$$
\int \psi_{\mathbf{l}}(\xi) \mu(d \xi)=0
$$


for each $\mathbf{l} \in \mathbb{R}^{d}$ it follows from a straightforward application of the ergodic theorem that the Stokes drift of the particle

$$
v:=\lim _{t \rightarrow+\infty} \frac{x_{t}}{t}, \quad \text { a.s. }
$$

equals zero. Also, due to the presence of the spectral gap for the tagged particle dynamics, one easily concludes, see e.g.[6], that the laws $x_{t} / \sqrt{t}$ converge to a normal law $N\left(0, \Sigma_{*}\right)$. $\Sigma_{*}=\left[\Sigma_{k, l}\right]$, called the self-diffusivity matrix of the environment, is given by

$$
\Sigma_{*}=(1-\rho) D_{0}-2 D_{*}(\rho)
$$

where $D_{0}=\left[D_{0}^{(k, l)}\right]$ with

$$
D_{0}^{(k, l)}=\sum_{x} p(x) x_{k} x_{l}
$$

is the co-variance matrix of the random walk with transition of probabilities $p(\cdot)$ and $D_{*}(\rho)=$ $\left[D_{*}^{(k, l)}(\rho)\right]$ can be calculated as follows. Thanks to $(2.9)$ for each $k=1, \ldots, d$ there exists a unique $\chi_{k}$ satisfying

$$
-\mathcal{L}_{0} \chi_{k}=\psi_{k} \quad \text { and } \int \chi_{k} d \mu=0 .
$$

Here $\psi_{k}:=\psi_{e_{k}}$, with $e_{k}:=\underbrace{(0, \ldots, 1, \ldots, 0)}_{k-\text { th position }}$. We have

$$
D_{*}^{k, l}(\rho)=\left(\psi_{k}, \chi_{l}\right)_{L^{2}(\mu)} .
$$

2.2. Tagged particle in the presence of an external field. We modify now the jump rates of the tagged particle. They will depend on a positive parameter $\alpha$ small enough and we denote them by $p(x ; \alpha)$. We assume that $p(x ; \alpha)$ depends smoothly on $\alpha$ and that $p(x ; 0)=p(x)$. We are particularly interested in the case $p(x ; \alpha)=p(x) \exp \{\alpha \mathbf{1} \cdot x\}$, with $\mathbf{l} \in \mathbb{R}^{d}$ and of length 1 . This choice corresponds to a perturbation given by an external field of strength $\alpha$ in the direction $\mathbf{l}$, cf. [13].

Since we are concerned only in the first order effects in $\alpha$, we can linearize $p(x ; \alpha)$ and with no loss of generality assume that it is of the form $p(x)+\alpha \ell(x)$ where $\ell: \mathbb{Z}^{d} \rightarrow \mathbb{R}$ is such that $p(x)+\alpha \ell(x) \geq 0$. We assume furthermore that the support of $\ell(\cdot)$ is contained in the support of $p(\cdot)$ (which, as we recall, is finite).

Define the operator

$$
\mathcal{A} g(\xi)=\sum_{x}(1-\xi(x)) \ell(x)\left[g\left(\tau_{x} \xi\right)-g(\xi)\right]
$$

A standard theory of Markov process, modifying slightly the approach taken in Chapter 1 of [11], guarantees that the operator

$$
\mathcal{L}_{\alpha}=\mathcal{L}_{0}+\alpha \mathcal{A}
$$

gives rise to a Markov process $\left(\xi_{t}^{(\alpha)}\right)_{t \geq 0}, \alpha \in \mathbb{R}$ whose generator restricted to $\mathcal{C}$ equals $\mathcal{L}_{\alpha}$. 
Now, in general, $\mu$ is not an invariant measure for $\xi_{t}^{(\alpha)}$ when $\alpha \neq 0$. Let $\left(Q_{\alpha}^{t}\right)_{t \geq 0}$ be the transition of probability semigroup that corresponds to $\xi_{t}^{(\alpha)}, t \geq 0$. Our first result concerning the existence of an invariant measure for this process can be stated as follows.

Theorem 2.2. There is $\alpha_{0}>0$ such that for any $0 \leq|\alpha|<\alpha_{0}$ the following statements are true.

1) There exists a unique invariant measure $\nu_{\alpha}$ for $\left(\xi_{t}^{(\alpha)}\right)_{t \geq 0}$ that is absolutely continuous w.r.t. $\mu$.

2) Let $f_{\alpha}:=d \nu_{\alpha} / d \mu$. Then, $f_{\alpha}>0, \mu$-a.s. In addition, there exist $g_{n} \in L^{2}(\mu), n \geq 1$, such that

$$
\left\|g_{n}\right\|_{L^{2}(\mu)} \leq M \alpha_{0}^{-n}, \quad \forall n \geq 1
$$

for some $M>0$ and

$$
f_{\alpha}=\mathbf{1}+\sum_{n=1}^{+\infty} \alpha^{n} g_{n}
$$

3) Suppose that the distribution of $\xi_{0}^{(\alpha)}$ possesses a square integrable density $g_{0}$ w.r.t. the unperturbed equilibrium measure $\mu$. Denote by $g_{t}$ the respective density of the law of $\xi_{t}^{(\alpha)}$. Then, there exists $\lambda>0$ such that

$$
\left\|g_{t}-f_{\alpha}\right\|_{L^{2}(\mu)} \leq\left\|g_{0}-f_{\alpha}\right\|_{L^{2}(\mu)} e^{-\lambda t}, \quad \forall t \geq 0 .
$$

Let $\mathbb{P}^{\alpha, \mu}$ denote the path measure on $D([0,+\infty) ; \Omega)$ corresponding to the process with the generator $\mathcal{L}_{\alpha}$ and the initial distribution $\mu$. By the ergodic theorem we conclude that

$$
v(\alpha):=\lim _{t \rightarrow+\infty} \frac{x_{t}}{t}=\int \psi^{(\alpha)}(\xi) \nu_{\alpha}(d \xi)
$$

where

$$
\psi^{(\alpha)}(\xi):=\sum_{z} z[p(z)+\alpha \ell(z)][1-\xi(z)] .
$$

Our next result is concerned with the asymptotic of $v(\alpha)$ for small $\alpha$. We let

$$
\phi_{\ell}(\xi):=\sum_{z}[\ell(z)-\ell(-z)][1-\xi(z)]
$$

Theorem 2.3. (Einstein's relation) The following asymptotic equality holds for $\alpha \ll 1$

$$
v_{k}(\alpha)=\left[(1-\rho) \sum_{z} z_{k} \ell(z)-\left(\phi_{\ell}, \chi_{k}\right)_{L^{2}(\mu)}\right] \alpha+o(\alpha), k=1, \ldots, d .
$$

In the particular case when $\ell(z)=\mathbf{l} \cdot z p(z)$ we have $\phi_{\ell}=2 \psi \cdot \mathbf{l}$ and we obtain

$$
v(\alpha)=\alpha \Sigma_{*} 1+o(\alpha) .
$$

where $\Sigma_{*}$ is given by (2.13).

The proofs of Theorems 2.2 and 2.3 are given in Section 3. 
Remark 2.4. (2.21) implies that Einstein's relation is valid only for particular perturbations, e.g. for the external constant field. In addition, even a mean zero perturbation $\ell(\cdot)$, i.e. $\sum_{z} z \ell(z)=0$, can cause the particle to pick up a non-zero drift. This can be seen in the following simple example.

Let $d=1, n \geq 2$ that we will choose large enough, and $p( \pm 1, \alpha)=1 / 2 \pm \alpha n, p( \pm n, \alpha)=$ $1 /\left(2 n^{2}\right) \mp \alpha$, and of course we take small perturbations $|\alpha| \leq 1 /\left(2 n^{2}\right)$. Then, the functional $\psi$ defined in (2.10) is given by $\psi(\xi)=1 / 2 A(\xi)+1 /(2 n) B(\xi)$, where

$$
A(\xi)=\xi(-1)-\xi(1) \quad \text { and } \quad B(\xi)=\xi(-n)-\xi(n)
$$

and $\chi=1 / 2 \chi_{A}+1 /(2 n) \chi_{B}$. Here $\chi_{A}, \chi_{B}$ are zero mean solutions of the Poisson equations $-\mathcal{L}_{0} \chi_{A}=A,-\mathcal{L}_{0} \chi_{B}=B$. Since $\|B\|_{L^{2}(\mu)}=\|A\|_{L^{2}(\mu)}=\sqrt{2 \rho(1-\rho)}$ the norms $\left\|\chi_{A}\right\|_{L^{2}(\mu)},\left\|\chi_{B}\right\|_{L^{2}(\mu)}$ can be bounded from above by $\sqrt{2 \rho(1-\rho)}$. On the other hand, here we have $\ell(n)=-\ell(-n)=1, \ell(1)=-\ell(-1)=-n, \ell(z)=0$ if otherwise. We have $\phi_{\ell}=2 n A-2 B$. For a sufficiently small $\alpha$ we have, according to (2.21),

$$
v(\alpha) / \alpha \approx-\left(\phi_{\ell}, \chi\right)_{L^{2}(\mu)}=-n\left(A, \chi_{A}\right)_{L^{2}(\mu)}+\frac{1}{n}\left(B, \chi_{B}\right)_{L^{2}(\mu)} .
$$

Now $\left(B, \chi_{B}\right)_{L^{2}(\mu)}$ is bounded from above by $2 \rho(1-\rho)$, while it is not difficult to prove that there exists a constant $C$ independent from $n$ and $\rho$ such that

$$
\left(A, \chi_{A}\right)_{L^{2}(\mu)}=\left(A,\left(-\mathcal{L}_{0}\right)^{-1} A\right) \geq C \rho(1-\rho)
$$

Indeed, to prove the lower bound (2.24) we use the variational expression

$$
\left(A,\left(-\mathcal{L}_{0}\right)^{-1} A\right)_{L^{2}(\mu)}=\sup _{\varphi}\{2(A, \varphi)-\mathcal{D}(\varphi)\}
$$

where $\mathcal{D}$ is defined by (2.6). Let $\varphi=\lambda A, \lambda>0$ is to be appropriately selected later on. A straightforward direct calculations produce, cf. $(2.7)-(2.8), \mathcal{D}_{0}(A)=\left(1+2 / n^{2}\right) \rho(1-\rho)$, $\mathcal{D}_{a d}(A)=2 \rho(1-\rho)$ and $\mathcal{D}_{\tau}(A)=\rho(1-\rho)\left(1+\rho+2 n^{-2}\right)$. Thus,

$$
\mathcal{D}(A)=\rho(1-\rho)\left(4+\frac{4}{n^{2}}+\rho\right) .
$$

According to (2.25)

$$
\left(A, \chi_{A}\right)_{L^{2}(\mu)} \geq 2 \lambda \rho(1-\rho)-\lambda^{2} \rho(1-\rho)\left(4+\frac{4}{n^{2}}+\rho\right) .
$$

Choosing $\lambda>0$ sufficiently small we can guarantee the bound on the utmost right hand side of (2.24). Observe that the effective velocity is negative. We can understand this fact in the following way. If the long jumps of length $n$ were not present, the effect of the perturbation would be to give the particle a positive effective velocity. This effective velocity would be, in the first order in $\alpha$, equal to the velocity of the particle without other particles present $(\rho=0)$, minus the autocorrelation term $\left(\phi_{\ell}, \chi\right)$ which represent the slowing down due to the blocking caused by the other particles. This blocking is due to the correlations of the stationary state: the tagged particle finds a higher density at its right than at its left. Now, if the jumps of order $n$ are allowed and the density of other particles is zero the tagged particle will have a null effective velocity, but with other particles at hand, the blocking effect will be still present and many of the short jumps to the right will be suppressed. This does 
not happen for the long jumps to the left, because of the fast decay of correlations of the stationary measure, so that the global effect is a negative velocity.

\section{The proofs of Theorems 2.2 And 2.3}

\subsection{The proof of parts 1) and 2) of Theorem 2.2.}

3.1.1. The existence of an invariant density. We make an Ansatz concerning the form of the invariant density. We shall be looking for the formal solution of the Fokker-Planck equation

$$
\mathcal{L}_{\alpha}^{*} f_{\alpha}=0
$$

among the elements of $L^{2}(\mu)$ of the form (2.18). (3.1) leads to a recursive relation on $g_{n}$ (recall that $\mathcal{L}_{0}$ is self-adjoint)

$$
\begin{gathered}
-\mathcal{A}^{*} \mathbf{1}=\mathcal{L}_{0} g_{1} \\
-\mathcal{A}^{*} g_{n}=\mathcal{L}_{0} g_{n+1}, \quad n \geq 1 .
\end{gathered}
$$

A simple calculation shows that

$$
\mathcal{A}^{*} \mathbf{1}(\xi)=-\phi_{\ell}(\xi)
$$

Since the left hand side of (3.2) is centered it has a unique solution $g_{1}$ satisfying $\int g_{1} d \mu=0$. By induction we conclude that if $g_{n}$ has been constructed in such a way that $\int g_{n} d \mu=0$ then clearly also $\int \mathcal{A}^{*} g_{n} d \mu=0$ and one can find a unique $g_{n+1}$ satisfying (3.3) that is centered. In addition, the spectral gap estimate allows us to write

$$
\left(g_{n}, \mathcal{A} g_{n+1}\right)_{L^{2}(\mu)}=-\left(\mathcal{L}_{0} g_{n+1}, g_{n+1}\right)_{L^{2}(\mu)} \geq \lambda_{0}\left\|g_{n+1}\right\|_{L^{2}(\mu)}^{2} .
$$

On the other hand we have the following.

Lemma 3.1. There exists a constant $C$ depending only on $p$ and $\ell$, such that

$$
\left|(f, \mathcal{A} g)_{L^{2}(\mu)}\right| \leq C\|f\|_{L^{2}(\mu)} \mathcal{D}^{1 / 2}(g),
$$

here $f, g$ are local functions.

Proof. Since there exists a constant $C$ such that $|\ell(x)| / p(x) \leq C / \sqrt{2}$, the left hand side of (3.5) can be estimated by

and (3.5) follows

$$
\begin{gathered}
\frac{1}{\sqrt{2}} C\|f\|_{L^{2}(\mu)}\left(\sum_{x \in S_{p}} \int[1-\xi(x)]\left|g\left(\tau_{x} \xi\right)-g(\xi)\right|^{2} \mu(d \xi)\right)^{1 / 2} \\
=C\|f\|_{L^{2}(\mu)} \mathcal{D}_{\tau}^{1 / 2}(g) \leq C\|f\|_{L^{2}(\mu)} \mathcal{D}^{1 / 2}(g)
\end{gathered}
$$

Continuing with the proof of Theorem 2.2 we note that (3.4) and (3.5) together imply that

$$
C\left\|g_{n}\right\|_{L^{2}(\mu)} \mathcal{D}^{1 / 2}\left(g_{n+1}\right) \geq \mathcal{D}\left(g_{n+1}\right)
$$

and in consequence

$$
C\left\|g_{n}\right\|_{L^{2}(\mu)} \geq \mathcal{D}^{1 / 2}\left(g_{n+1}\right) \geq \lambda_{0}^{1 / 2}\left\|g_{n+1}\right\|_{L^{2}(\mu)}, \quad \forall n \geq 1 .
$$

(3.6) clearly implies (2.17) for a suitable choice of $\alpha_{0}$. Note that for $\alpha$ as the statement of the theorem the series given on the right hand side of (2.18) converges in $L^{2}(\mu)$. The proof 
that $f_{\alpha}$ is not only a formal but also the "true" solution to the Fokker-Planck equation shall be presented after the proof of Lemma 3.2 below.

3.1.2. Positivity. First we show that $f_{\alpha}$ must be nonnegative. Denote by $f_{\alpha}^{+}, f_{\alpha}^{-}$the positive and negative parts of $f_{\alpha}$. Suppose that they are both nontrivial. Then $f_{+}:=f_{\alpha}^{+} /\left\|f_{\alpha}^{+}\right\|_{L^{1}(\mu)}$, $f_{-}:=f_{\alpha}^{-} /\left\|f_{\alpha}^{-}\right\|_{L^{1}(\mu)}$ should also be invariant densities. Indeed, to see the above it suffices only to show that

$$
\int \mathbf{1}_{A} f_{\alpha}^{ \pm} d \mu=\int Q_{\alpha}^{t} \mathbf{1}_{A} f_{\alpha}^{ \pm} d \mu
$$

for all $A \in \mathcal{B}(\Omega)$ and $t \geq 0$. Since $f_{\alpha}^{+} \geq f_{\alpha}$, the positivity of $Q_{\alpha}^{t}$ implies that for any $A \in \mathcal{B}(\Omega)$ we have $Q_{\alpha}^{t} \mathbf{1}_{A} f_{\alpha}^{+} \geq Q_{\alpha}^{t} \mathbf{1}_{A} f_{\alpha}$, hence

$$
\int Q_{\alpha}^{t} \mathbf{1}_{A} f_{\alpha}^{+} d \mu \geq \int Q_{\alpha}^{t} \mathbf{1}_{A} f_{\alpha} d \mu=\int \mathbf{1}_{A} f_{\alpha} d \mu
$$

Let $A_{*}:=\operatorname{supp} f_{\alpha}^{+}$and $A \in \mathcal{B}(\Omega)$. Using the fact that $Q_{\alpha}^{t}$ is monotone we have $Q_{\alpha}^{t} \mathbf{1}_{A} \geq$ $Q_{\alpha}^{t}\left(\mathbf{1}_{A \cap A_{*}}\right)$ and therefore

$$
\int Q_{\alpha}^{t} \mathbf{1}_{A} f_{\alpha}^{+} d \mu \geq \int Q_{\alpha}^{t}\left(\mathbf{1}_{A \cap A_{*}}\right) f_{\alpha}^{+} d \mu \stackrel{(3.8)}{\geq} \int \mathbf{1}_{A} \mathbf{1}_{A_{*}} f_{\alpha} d \mu=\int \mathbf{1}_{A} f_{\alpha}^{+} d \mu .
$$

The inequality in (3.9) can be in fact strengthen to an equality. Indeed, from (3.9) applied to $A^{c}$ we have $\int Q_{\alpha}^{t} \mathbf{1}_{A^{c}} f_{\alpha}^{+} d \mu \geq \int \mathbf{1}_{A^{c}} f_{\alpha}^{+} d \mu$ and since $Q^{t} \mathbf{1}=\mathbf{1}$ these facts together of course imply $\int Q_{\alpha}^{t} \mathbf{1}_{A} f_{\alpha}^{+} d \mu=\int \mathbf{1}_{A} f_{\alpha}^{+} d \mu$ for all $A \in \mathcal{B}(\Omega)$, which proves that $f_{\alpha}^{+}$is invariant. The invariance of $f_{\alpha}^{-}$can be shown in an identical fashion. The above argument shows that $\mathcal{L}_{\alpha}^{*} f_{ \pm}=0$. On the other hand, thanks to the uniqueness result on the solutions of the Fokker-Planck equation (see Lemma 3.2 below), we must have $f_{-}=f_{+}$, which leads to a contradiction.

We proceed with the proof of strict positivity. Let $A_{*}:=\operatorname{supp} f_{\alpha}$. We have $\mu\left(A_{*}\right)>0$ and suppose that $A_{*}^{c}:=\Omega \backslash A_{*}$ is of positive $\mu$-probability. Then,

$$
\left(Q_{\alpha}^{t} \mathbf{1}_{A_{*}^{c}}, f_{\alpha}\right)_{L^{2}(\mu)}=0 \quad \text { for all } t \geq 0 .
$$

Denote by $\mathbb{P}^{\alpha, \eta}$ the path measure on $D([0,+\infty) ; \Omega)$ associated to the process with the generator $\mathcal{L}_{\alpha}$ and the initial configuration $\eta$. Since the processes corresponding to different values of $\alpha$ have the same allowed jumps the path measures $\mathbb{P}^{\alpha, \eta}$ and $\mathbb{P}^{0, \eta}$ are equivalent when considered over $D([0, T] ; \Omega)$ for each $T>0$, see e.g. [9] p. 326. Using this notation we can recast (3.10) in the form

$$
\int\left[\int \mathbf{1}_{A_{*}^{c}}(\xi(t)) \mathbb{P}^{\alpha, \eta}(d \xi)\right] f_{\alpha}(\eta) \mu(d \eta)=0 .
$$

(3.11) in turn implies that

$$
\int \mathbf{1}_{A_{*}^{c}}(\xi(t)) \mathbb{P}^{\alpha, \eta}(d \xi)=\int \mathbf{1}_{A_{*}^{c}}(\xi(t)) \mathbb{P}^{0, \eta}(d \xi)=0
$$


for all $\eta \in A_{*}$. Hence,

$$
\left(P^{t} \mathbf{1}_{A_{*}^{c}}, \mathbf{1}_{A_{*}}\right)_{L^{2}(\mu)}=\int_{A_{*}}\left[\int \mathbf{1}_{A_{*}^{c}}(\xi(t)) \mathbb{P}^{0, \eta}(d \xi)\right] \mu(d \eta)=0
$$

so $P^{t} \mathbf{1}_{A_{*}^{c}} \leq \mathbf{1}_{A_{*}^{c}}$. By reversibility we also get $P^{t} \mathbf{1}_{A_{*}} \leq \mathbf{1}_{A_{*}}$. These two conditions however contradict ergodicity of $\mu$.

3.1.3. Uniqueness of an invariant measure. We prove the following lemma.

Lemma 3.2. The operator $K:=\mathcal{L}_{0}^{-1} \mathcal{A}^{*}$ is well defined on the space $\mathcal{C}_{0}$ of zero $\mu$-average local functions. It extends to a bounded operator $K: L_{0}^{2}(\mu) \rightarrow L_{0}^{2}(\mu)$ and there exists $\alpha_{0}>0$ such that $I+\alpha K$ is a linear isomorphism of $L_{0}^{2}(\mu)$ for $|\alpha| \leq \alpha_{0}$. Moreover,

$$
(I+\alpha K) f=\mathcal{L}_{0}^{-1} g, \quad \text { for some } g \in L_{0}^{2}(\mu)
$$

iff $f \in D\left(\mathcal{L}_{\alpha}^{*}\right)$ and

$$
\mathcal{L}_{\alpha}^{*} f=g .
$$

Proof. Let $f \in \mathcal{C}_{0}$. Substituting $g=1$ in (3.5) we conclude that $\int \mathcal{A}^{*} f d \mu=0$, hence $\mathcal{A}^{*} f$ belongs to the range of $\mathcal{L}_{0}$. Indeed, thanks to the spectral gap condition we can always write $\mathcal{L}_{0}^{-1} \mathcal{A}^{*} f=-\int_{0}^{\infty} Q^{t} \mathcal{A}^{*} f d t$. Furthermore, for any $g \in L_{0}^{2}(\mu)$ we have

$$
\begin{gathered}
\left|\left(\mathcal{L}_{0}^{-1} \mathcal{A}^{*} f, g\right)_{L^{2}(\mu)}\right|=\left|\left(\mathcal{A}^{*} f, \mathcal{L}_{0}^{-1} g\right)_{L^{2}(\mu)}\right| \leq C\|f\|_{L^{2}(\mu)} \mathcal{D}^{1 / 2}\left(\mathcal{L}_{0}^{-1} g\right) \\
=C\|f\|_{L^{2}(\mu)}\left(\left(-\mathcal{L}_{0}\right)^{-1} g, g\right)_{L^{2}(\mu)}^{1 / 2} \leq \frac{C}{\sqrt{\lambda_{0}}}\|f\|_{L^{2}(\mu)}\|g\|_{L^{2}(\mu)}
\end{gathered}
$$

and boundedness of $K$ follows. The fact that $I+\alpha K$ is a linear isomorphism for a sufficiently small $\alpha$ can be concluded via a standard perturbation argument. Note also that for any $h \in \mathcal{C}_{0}$ we have

$$
((I+\alpha K) f, h)_{L^{2}(\mu)}=\left(\mathcal{L}_{\alpha}^{*} f, \mathcal{L}_{0}^{-1} h\right)_{L^{2}(\mu)} .
$$

Suppose now that (3.12) holds. Then, for any $h \in \mathcal{C}_{0}$ we have

$$
\begin{aligned}
\left(f, \mathcal{L}_{\alpha} h\right)_{L^{2}(\mu)} & =\left(f, \mathcal{L}_{0} h+\alpha \mathcal{A} h\right)_{L^{2}(\mu)}=\left((I+\alpha K) f, \mathcal{L}_{0} h\right)_{L^{2}(\mu)} . \\
& =\left(\mathcal{L}_{0}^{-1} g, \mathcal{L}_{0} h\right)_{L^{2}(\mu)}=(g, h)_{L^{2}(\mu)},
\end{aligned}
$$

which implies that $f \in D\left(\mathcal{L}_{\alpha}^{*}\right)$ and (3.13) holds (recall that $f \in D\left(\mathcal{L}_{\alpha}^{*}\right)$ iff the linear functional $g \mapsto\left(f, \mathcal{L}_{\alpha} g\right)_{L^{2}(\mu)}$ extends in a bounded way to entire $\left.L^{2}(\mu)\right)$.

The converse follows from the fact that (3.13) implies

$$
\begin{gathered}
(g, h)_{L^{2}(\mu)}=\left(\mathcal{L}_{\alpha}^{*} f, h\right)_{L^{2}(\mu)}=\left(f, \mathcal{L}_{0} h+\alpha \mathcal{A} h\right)_{L^{2}(\mu)} \\
\stackrel{(3.14)}{=}\left((I+\alpha K) f, \mathcal{L}_{0} h\right)_{L^{2}(\mu)}, \quad \forall h \in \mathcal{C}_{0} .
\end{gathered}
$$

We shall finish the proof that $f_{\alpha}$ constructed in the existence part of the proof satisfies the Fokker-Planck equation. Let $\tilde{f}_{\alpha}=f_{\alpha}-\mathbf{1}$. It is elementary to observe from the construction of $f_{\alpha}$ that

$$
(I+\alpha K) \tilde{f}_{\alpha}=-\alpha \mathcal{L}_{0}^{-1} \mathcal{A}^{*} \mathbf{1}
$$


hence according to Lemma 3.2 we have $f_{\alpha} \in D\left(\mathcal{L}_{\alpha}^{*}\right)$ and (3.1) holds

3.2. The proof of part 3) of Theorem 2.2. It suffices to show inequality (2.19) under the assumption that $g_{0} \in D\left(\mathcal{L}_{0}+\alpha \mathcal{A}^{*}\right)$. Then, obviously $g_{t} \in D\left(\mathcal{L}_{0}+\alpha \mathcal{A}^{*}\right)$ and, thanks to the fact that $\mathcal{A}^{*}$ is bounded we can estimate

$$
\begin{gathered}
\frac{d}{d t}\left\|g_{t}-f_{\alpha}\right\|_{L^{2}(\mu)}^{2}=2\left(\left(\mathcal{L}_{0}+\alpha \mathcal{A}^{*}\right)\left(g_{t}-f_{\alpha}\right), g_{t}-f_{\alpha}\right)_{L^{2}(\mu)} \\
=-2 \mathcal{D}\left(g_{t}-f_{\alpha}\right)+\alpha\left(\mathcal{A}^{*}\left(g_{t}-f_{\alpha}\right), g_{t}-f_{\alpha}\right)_{L^{2}(\mu)} \\
\stackrel{(2.9)}{\leq}-2 \mathcal{D}\left(g_{t}-f_{\alpha}\right)+\lambda_{0}^{-1 / 2} \alpha\left\|\mathcal{A}^{*}\right\| \mathcal{D}^{1 / 2}\left(g_{t}-f_{\alpha}\right)\left\|g_{t}-f_{\alpha}\right\|_{L^{2}(\mu)} \leq \\
\stackrel{(2.9)}{\leq}-\left(2-\frac{C \alpha}{\lambda_{0}^{1 / 2}}\right) \mathcal{D}\left(g_{t}-f_{\alpha}\right) \leq-\lambda_{0}\left(2-\frac{C \alpha}{\lambda_{0}^{1 / 2}}\right)\left\|g_{t}-f_{\alpha}\right\|_{L^{2}(\mu)}^{2} .
\end{gathered}
$$

When $g_{0}=f_{\alpha}$ and $|\alpha|<2 \lambda_{0}^{1 / 2} / C$ the conclusion follows from a standard application of Gronwall's inequality.

3.3. The proof of Theorem 2.3. Note that according to (2.20) and (2.18) the $k$-th component of the particle's Stokes drift $v(\alpha)$ equals, up to a term of order $o(1)$,

$$
\begin{aligned}
(1-\rho) \sum_{z} z_{k} \alpha \ell(z)+ & \left(g_{1}, \alpha \psi_{k}\right)_{L^{2}(\mu)} \stackrel{(3.2)}{=}(1-\rho) \sum_{z} z_{k} \alpha \ell(z)-\alpha\left(\mathcal{A}^{*} \mathbf{1}, \mathcal{L}_{0}^{-1} \psi_{k}\right)_{L^{2}(\mu)} \\
& =(1-\rho) \sum_{z} z_{k} \alpha \ell(z)-\alpha\left(\phi_{\ell}, \chi_{k}\right)_{L^{2}(\mu)}
\end{aligned}
$$

and (2.21) follows.

\section{A general Model and some more examples}

In the previous section we have applied the perturbative approach to the exclusion model with adsorption-desorption of particles. We will describe here a more general set-up for theorems 2.2 and 2.3. In particular we will discuss the role of reversibility. Some of the ideas exposed in this section are inspired by [10] and [14].

4.1. Environment dynamics. Let $\Omega$ be a Polish metric space of the environment configurations. We consider then an $\Omega$-valued, cadlag Markov process $\left(\xi_{t}\right)_{t \geq 0}$ and suppose that

R) $\mu$ is an invariant, ergodic measure for this process, i.e. the corresponding $L^{2}(\mu)$-transition probability semigroup $Q^{t}: L^{2}(\mu) \rightarrow L^{2}(\mu), t \geq 0$ satisfy

$$
\left(Q^{t} f, \mathbf{1}\right)_{L^{2}(\mu)}=(f, \mathbf{1})_{L^{2}(\mu)}, \quad \forall f \in L^{2}(\mu), t \geq 0
$$

and $Q^{t} f=f$ for all $t \geq 0$ iff $f$ is constant. This semigroup is $C_{0}$-continuous and we denote by $\mathcal{L}_{0}: D\left(\mathcal{L}_{0}\right) \rightarrow L^{2}(\mu)$ its generator.

Observe that we do not assume that this generator is symmetric in $L^{2}(\mu)$. We denote by $\mathcal{E}_{\mathcal{L}_{0}}(f, g)=\left(-\mathcal{L}_{0} f, g\right)_{L^{2}(\mu)}$ the corresponding Dirichlet form (in general non-symmetric) defined for appropriate $f, g \in D\left(\mathcal{E}_{\mathcal{L}_{0}}\right)$ - the domain of the form. 
SG) The semigroup $\left(Q^{t}\right)_{t \geq 0}$ satisfies the spectral gap condition, i.e. there exists $\lambda_{0}>0$, such that

$$
\lambda_{0}\|f\|_{L^{2}(\mu)}^{2} \leq \mathcal{E}_{\mathcal{L}_{0}}(f, f), \quad \forall f \in D\left(\mathcal{E}_{\mathcal{L}_{0}}\right) \cap L_{0}^{2}(\mu) .
$$

4.2. Perturbations and the existence of a steady state. We suppose that for a given $\alpha,\left(\xi_{t}^{(\alpha)}\right)_{t \geq 0}$ is another $\Omega$-valued, cadlag, Markov process. Let $Q_{\alpha}^{t}: B_{b}(\Omega) \rightarrow B_{b}(\Omega), t \geq 0$ be its corresponding transition of probability. Here $B_{b}(\Omega)$ denotes the space of all bounded and measurable functions. Note that we do not require this semigroup to be $C_{0}$-continuous.

We assume that

C) there exists $\mathcal{C}$, a linear subspace of $B_{b}(\Omega)$, containing 1 that is a core of both $\mathcal{L}_{0}$ and $\mathcal{L}_{0}^{*}$, the adjoint operator to $\mathcal{L}_{0}$ w.r.t. the scalar product $(\cdot, \cdot)_{L^{2}(\mu)}$. In addition, we assume that there exists a certain $\alpha_{1}>0$ for which $Q_{\alpha}^{t}(\mathcal{C}) \subseteq \mathcal{C}$ for all $t \geq 0,|\alpha| \leq \alpha_{1}$ and $\left(Q_{\alpha}^{t} f-f\right) / t$ tends $L^{2}(\mu)$-weakly to $\mathcal{L}_{\alpha} f$, as $t \rightarrow 0+$ for all $f \in \mathcal{C}$.

$\mathrm{SC})$ The operator $\mathcal{L}_{\alpha}: \mathcal{C} \rightarrow L^{2}(\mu)$ can be written in the form

$$
\mathcal{L}_{\alpha} f=\mathcal{L}_{0} f+\alpha \mathcal{A}_{\alpha} f, \quad f \in \mathcal{C},
$$

where both $\mathcal{A}_{\alpha}$ and $\mathcal{A}_{\alpha}^{*}$ are operators that satisfy the sector condition in the following sense

$$
\left|\left(f, \mathcal{A}_{\alpha} g\right)_{L^{2}(\mu)}\right| \leq C \mathcal{E}_{\mathcal{L}_{0}}^{1 / 2}(g, g)\|f\|_{L^{2}(\mu)}, \quad\left|\left(f, \mathcal{A}_{\alpha}^{*} g\right)_{L^{2}(\mu)}\right| \leq C \mathcal{E}_{\mathcal{L}_{0}}^{1 / 2}(g, g)\|f\|_{L^{2}(\mu)}
$$

for all $f, g \in \mathcal{C}$ and some $C>0$.

In particular SC) implies that $\mathcal{A}_{\alpha}$ is closable and $D\left(\mathcal{L}_{0}\right) \subseteq D\left(\overline{\mathcal{A}}_{\alpha}\right)$. In what follows we denote the closure of $\mathcal{A}_{\alpha}$ by the same symbol.

Let $g_{1}^{(\alpha)}$ be the unique $\mu$-zero mean solution of the equation $-\mathcal{A}_{\alpha}^{*} \mathbf{1}=\mathcal{L}_{0}^{*} g_{1}^{(\alpha)}$. The existence and uniqueness of such a $g_{1}^{(\alpha)}$ follows easily from the fact that $\mathcal{L}_{0}^{*}$ is a generator of the dual semigroup $\left(Q^{t}\right)^{*}, t \geq 0$ in $L^{2}(\mu)$ which also satisfies the spectral gap condition with the same constant $\lambda_{0}$. We assume the following continuity condition:

$$
\lim _{\alpha \rightarrow 0} g_{1}^{(\alpha)}=g_{1}:=g_{1}^{(0)} .
$$

The following result concerning the existence of a steady state for the process, that is absolutely continuous with respect to $\mu$, can be obtained using essentially the same argument (with some trivial adjustments due to non-symmetry of $\mathcal{L}_{0}$ ) as the one in the proof of Theorem 2.2

Theorem 4.1. Under the assumptions $R), S G), C), S C$ ) and $P$ ) there exists $\alpha_{0}>0$ such that for any $|\alpha| \leq \alpha_{0}$ there is a unique invariant measure $\nu_{\alpha}$ for $\left(\xi_{t}^{(\alpha)}\right)_{t \geq 0}$ that is absolutely continuous w.r.t. $\mu$. Let $f_{\alpha}:=d \nu_{\alpha} / d \mu$. Then, $f_{\alpha} \in L^{2}(\mu)$ and there exist $g_{n}:\left[-\alpha_{0}, \alpha_{0}\right] \rightarrow$ $L^{2}(\mu), n \geq 1$, such that

$$
\left\|g_{n}^{(\alpha)}\right\|_{L^{2}(\mu)} \leq M \alpha_{0}^{-n}, \quad \forall n \geq 1,|\alpha| \leq \alpha_{0}
$$

for some $M$ independent of $\alpha, n$ and

$$
f_{\alpha}=\mathbf{1}+\sum_{n=1}^{+\infty} \alpha^{n} g_{n}^{(\alpha)} .
$$


Moreover, part 3) of the statement of Theorem 2.2 holds also in this case.

Observe that, since $g_{1}^{(\alpha)}$ is the solution of the equation $\mathcal{A}_{\alpha}^{*} 1=-\mathcal{L}_{0}^{*} g_{1}^{(\alpha)}$, we have $\int \mathcal{A}_{\alpha} g_{1}^{(\alpha)} d \mu=$ $\mathcal{E}_{\mathcal{L}_{0}}\left(g_{1}^{(\alpha)}, g_{1}^{(\alpha)}\right)$.

4.3. Tracer dynamics. Let us first consider the unperturbed case. The position of the tracer is usually given by an additive functional $x_{t}=\left(x_{1, t}, \ldots, x_{d, t}\right) \in \mathbb{R}^{d}$ of the environment process $\left(\xi_{t}\right)_{t \geq 0}$. With no change to the argument we could admit $x_{t} \in \mathbb{Z}^{d}$. We assume that

T-1 there exists a vector valued function $\psi=\left(\psi_{1}, \ldots, \psi_{d}\right)$ with $\psi_{p} \in L^{2}(\mu), p=1, \ldots, d$

(the so-called mean forward velocity) with null $\mu$-average, such that

$$
x_{t}=\int_{0}^{t} \psi\left(\xi_{s}\right) d s+M_{t}
$$

where $\left(M_{t}\right)_{t \geq 0}$ is a square integrable vector valued martingale with respect to the natural filtration of the process $\left(\xi_{t}\right)_{t \geq 0}$ satisfying $M_{0} \equiv 0$.

T-2 the joint quadratic variation of the vector martingale $\left(M_{t}\right)_{t \geq 0}$ is given by

$$
\left\langle M_{p}, M_{q}\right\rangle_{t}=\int_{0}^{t} \theta_{p, q}\left(\xi_{s}\right) d s
$$

Since the martingale is square integrable we must have $\theta_{p, q} \in L^{1}(\mu)$.

T-3 The $\mathbb{R}^{d} \times \Omega$-valued joint process $\left(x_{t}, \xi_{t}\right)_{t \geq 0}$ is Markovian and denote its generator by $\mathfrak{L}_{0}$.

T-4 $x_{t}$ is an anti-symmetric functional of $\left\{\xi_{s}\right\}_{0 \leq s \leq t}$ with respect the time reversal transformation $R_{t}(\xi .)_{s}=\xi_{t-s}$ for $0 \leq s \leq t$. This means that, denoting $x_{t}=X_{t}(\xi$.$) , where X_{t}(\cdot)$ is $\mathcal{F}_{t}:=\sigma\left(\xi_{s} ; 0 \leq s \leq t\right)$-measurable, we have $X_{t}\left(R_{t}(\xi).\right)=-X_{t}(\xi)=.-x_{t}$.

Since the $\xi_{t}$ process is autonomous, we have that $\mathfrak{L}_{0}$, when restricted to function of the form $f(x, \xi)=f(\xi)$, coincides with $\mathcal{L}_{0}$ for any $f \in D\left(\mathcal{L}_{0}\right)$. The conditions $\mathbf{T}-\mathbf{3}$ and $\mathbf{T}-\mathbf{4}$ are automatically satisfied in all examples we are interested. We recall that, in the stationary state induced by $\mu$, the process $\xi_{s}^{*}=R_{t}(\xi .)_{s}$ is Markovian with the generator $\mathcal{L}_{0}^{*}$.

T-5 There exists a vector valued function $\psi^{*}=\left(\psi_{1}^{*}, \ldots, \psi_{d}^{*}\right)$ with $\psi_{p}^{*} \in L^{2}(\mu), p=1, \ldots, d$ (the mean backward velocity) with null $\mu$-average, such that

$$
X_{s}\left(\xi^{*}\right)=\int_{0}^{s} \psi^{*}\left(\xi_{\tau}^{*}\right) d \tau+M_{s}^{*}, \quad 0 \leq s \leq t
$$

where $\left(M_{s}^{*}\right)_{0}$ is a square integrable vector valued martingale with respect to the filtration of the process $\left(\xi_{s}^{*}\right)_{0 \leq s \leq t}$ satisfying $M_{0}^{*} \equiv 0$.

Observe that $X_{t}\left(\xi^{*}\right)=-x_{t}$.

Let us define $U_{p}(x)=x_{p}, p=1, \ldots, d$. By (4.6) and (4.7) we have

$$
\psi_{p}=\mathfrak{L}_{0} U_{p}, \quad \theta_{p, q}=\mathfrak{L}_{0}\left(U_{p} U_{q}\right)-U_{p} \mathfrak{L}_{0} U_{q}-U_{q} \mathfrak{L}_{0} U_{p} .
$$

Observe that the above functions do not depend on $x$. 
Thanks to hypothesis SG), there exists a unique zero mean solution $\chi_{p} \in L^{2}(\mu)$ to the equation

$$
-\mathcal{L}_{0} \chi_{p}=\psi_{p}
$$

Using (4.9) and the stationarity of $\mu$, we can compute the covariance matrix of $x_{t}$ in the stationary state $\mu$ as

$$
\begin{aligned}
\mathbb{E}_{\mu}\left(x_{p, T} x_{q, T}\right) & =\mathbb{E}_{\mu}\left[\int_{0}^{T}\left(\mathfrak{L}_{0} U_{p} U_{q}\right)\left(x_{t}, \xi_{t}\right) d t\right] \\
& =T \int \theta_{p, q} d \mu+\int_{0}^{T} \mathbb{E}_{\mu}\left(U_{p}\left(x_{t}\right) \psi_{q}\left(\xi_{t}\right)+U_{q}\left(x_{t}\right) \psi_{p}\left(\xi_{t}\right)\right) d t
\end{aligned}
$$

Now by assumptions $\mathbf{T}-\mathbf{4}$ and $\mathbf{T}-\mathbf{5}$ we have

$$
\begin{aligned}
\mathbb{E}_{\mu}\left(U_{p}\left(x_{t}\right) \psi_{q}\left(\xi_{t}\right)\right) & =-\mathbb{E}_{\mu}^{*}\left(U_{p}\left(X_{t}\left(\xi^{*} \cdot\right)\right) \psi_{q}\left(\xi_{0}^{*}\right)\right)=-\int_{0}^{t} \mathbb{E}_{\mu}^{*}\left(\psi_{p}^{*}\left(\xi_{s}^{*}\right) \psi_{q}\left(\xi_{0}^{*}\right)\right) d s \\
& =-\int_{0}^{t}\left(\psi_{p}^{*}, Q^{s} \psi_{q}\right)_{L^{2}(\mu)}
\end{aligned}
$$

We have then that the asymptotic covariance matrix exists and is given by

$$
\Sigma_{*}^{p, q}=\lim _{t \rightarrow \infty} \frac{1}{t} \mathbb{E}\left(x_{p, t} x_{q, t}\right)=\int\left(\theta_{p, q}-\psi_{p}^{*} \chi_{q}-\psi_{q}^{*} \chi_{p}\right) d \mu
$$

We are now ready for introducing the perturbed process obtained by the action of an external constant force. The way to do that is not always evident in such a general context. A natural assumption is that the path measure $Q^{(\alpha)}$ obtained by such perturbation is absolutely continuous, locally in time, with respect to the original measure. This suggest to define the path measure $Q^{(\alpha)}$ by the Radon-Nikodym derivative with respect to the measure $Q^{(0)}$ associated to the Markov process generated by $\mathfrak{L}_{0}$. This is given by the following Girsanov formula

$$
\left.\frac{d Q_{0, \eta}^{(\alpha)}}{d Q_{0, \eta}^{(0)}}\right|_{\mathcal{F}_{T}}=\exp \left\{\alpha U_{\mathbf{l}}\left(x_{T}\right)-\int_{0}^{T} \mathrm{e}^{-\alpha U_{\mathbf{l}}\left(x_{s}\right)}\left(\mathfrak{L}_{0} \mathrm{e}^{\alpha U_{\mathbf{l}}}\right)\left(x_{s}, \xi_{s}\right) d s\right\} .
$$

where $U_{\mathbf{l}}(x)=\mathbf{l} \cdot x$ and $\mathbf{l}=\left(\ell_{1}, \ldots, \ell_{d}\right) \in \mathbb{S}^{d-1}$ is a fixed direction.

It follows that $Q^{(\alpha)}$ is the path measure of a Markov process $\left(x_{t}^{(\alpha)}, \xi_{t}^{(\alpha)}\right)$ with generator (cf. eg. [9], appendix 1)

$$
\mathfrak{L}_{\alpha} H(x, \xi)=\mathrm{e}^{-\alpha U_{\mathbf{l}}(x)} \mathfrak{L}_{0}\left(\mathrm{e}^{\alpha U_{1}} H\right)(x, \xi)-\mathrm{e}^{-\alpha U_{\mathbf{l}}(x)}\left(H \mathfrak{L}_{0}\left(\mathrm{e}^{\alpha U_{1}}\right)\right)(x, \xi) .
$$

A standard argument, by approximating $U_{\mathbf{l}}$ by bounded functions, will make sense of (4.14) and (4.15). We decompose then $\mathfrak{L}_{\alpha}$ as

$$
\mathfrak{L}_{\alpha}=\mathfrak{L}_{0}+\alpha \mathfrak{A}_{\alpha}
$$

Let us add the further assumption 
T-6 When applied to functions $H(x, \xi)=H(\xi), \mathfrak{L}_{\alpha} H$ does not depend on $x$ at it coincides to the operator

$$
\mathcal{L}_{\alpha}=\mathcal{L}_{0}+\alpha \mathcal{A}_{\alpha}
$$

In the typical examples, T-6 is a consequence of some properties of translation invariance of the dynamics.

It follows from condition T-6 that, as in the unperturbed case, $\xi_{t}^{(\alpha)}$ is an autonomous Markov process.

Formally expanding $\mathcal{A}_{\alpha}$ in $\alpha$ we have

$$
\begin{aligned}
\mathcal{A}_{\alpha} H(\xi) & =\mathfrak{L}_{0}\left(U_{\mathbf{l}} H\right)(x, \xi)-U_{\mathbf{l}}(x)\left(\mathcal{L}_{0} H\right)(\xi)-H(\xi) \psi(\xi) \cdot \mathbf{l}+\mathcal{O}_{H}(\alpha ; x, \xi) \\
& =\mathfrak{L}_{0}\left(U_{\mathbf{l}} H\right)(0, \xi)-H(\xi) \psi(\xi) \cdot \mathbf{l}+\mathcal{O}_{H}(\alpha ; \xi),
\end{aligned}
$$

where $H$ depending only on $\xi$ belongs to $\mathcal{C}$ and $\mathcal{O}_{H}(\alpha ; \xi):=\mathcal{O}_{H}(\alpha ; 0, \xi)$.

T-7 We assume that $\mathcal{A}_{\alpha}$ satisfies condition SC) (cf subsection 4.2) and that $\int \mathcal{O}_{H}(\alpha ; \xi) d \mu(\xi) \rightarrow$ 0 as $\alpha \rightarrow 0$. Under this perturbed dynamics the position of the tracer is given by

$$
x_{t}^{(\alpha)}=\int_{0}^{t} \psi\left(\xi_{s}^{(\alpha)}, \alpha\right) d s+M_{t}^{(\alpha)}
$$

where $\psi(\xi, \alpha)=\left(\psi_{1}(\xi, \alpha), \ldots, \psi_{d}(\xi, \alpha)\right)$ and $\psi_{p}(\xi, \alpha):=\mathfrak{L}_{\alpha} U_{p}(\xi)$ and $\left(M_{t}^{(\alpha)}\right)$ is a square integrable martingale with respect to the natural filtration of the process $\left(\xi_{t}^{(\alpha)}\right)_{t \geq 0}$ satisfying $M_{0}^{(\alpha)} \equiv 0$.

Expanding $\psi(\xi, \alpha)$ with respect to $\alpha$ one obtain

$$
\psi_{p}(\xi, \alpha)=\psi_{p}+\alpha \sum_{q=1}^{d} \theta_{p, q}(\xi) \ell_{q}+\alpha \mathcal{O}_{p}(\xi, \alpha), \quad|\alpha| \ll 1, p=1, \ldots, d .
$$

T-8 We assume that $\int O_{p}(\xi, \alpha) d \mu(\xi) \rightarrow 0$ as $\alpha \rightarrow 0$,.

By Theorem 4.1 the asymptotic velocity can be computed and it equals

$$
v(\alpha):=\lim _{t \rightarrow+\infty} \frac{x_{t}^{(\alpha)}}{t}=\int \psi(\xi, \alpha) f_{\alpha}(\xi) d \mu(\xi)
$$

By the expansions (4.5) and (4.20) we have

$$
\begin{array}{r}
v_{p}(\alpha)=\alpha\left(\sum_{q=1}^{d} \int \theta_{p, q} \ell_{q} d \mu+\int \psi_{p} g_{1}^{(\alpha)} d \mu\right)+o(\alpha) \\
=\alpha\left(\sum_{q=1}^{d} \int \theta_{p, q} \ell_{q} d \mu-\int \chi_{p} \mathcal{L}_{0}^{*} g_{1}^{(\alpha)} d \mu\right)+o(\alpha)
\end{array}
$$


Recall that $\mathcal{L}_{0}^{*} g_{1}^{(\alpha)}=-\mathcal{A}_{\alpha}^{*} \mathbf{1}$, hence

$$
-\int \chi_{p} \mathcal{L}_{0}^{*} g_{1}^{(\alpha)} d \mu=\int \mathcal{A}_{\alpha} \chi_{p} d \mu \stackrel{(4.18)}{=} \sum_{q=1}^{d} \ell_{q}\left(\int \mathfrak{L}_{0}\left(U_{q} \chi_{p}\right) d \mu-\int \chi_{p} \psi_{q} d \mu\right)+O_{\chi_{p}}(\alpha) .
$$

By a similar computation as in (4.12) we have

$$
\begin{aligned}
\int \mathfrak{L}_{0}\left(U_{q} \chi_{p}\right)(0, \xi) d \mu(\xi) & =\frac{d}{d t} \mid t=0 \\
& \stackrel{(4.8)}{=}-\int \psi_{q}^{*}(\xi) \chi_{p}(\xi) d \mu(\xi)
\end{aligned}
$$

so we obtain

$$
\begin{aligned}
\lim _{\alpha \rightarrow 0} \frac{v_{p}(\alpha)}{\alpha} & =\sum_{q=1}^{d} \ell_{q} \int\left(\theta_{p, q}-\psi_{q}^{*} \chi_{p}-\psi_{q} \chi_{p}\right) d \mu \\
& =\sum_{q=1}^{d} \ell_{q} \Sigma_{*}^{p, q}+\sum_{q=1}^{d} \ell_{q} \int\left(\psi_{p}^{*} \chi_{q}-\psi_{q} \chi_{p}\right) d \mu .
\end{aligned}
$$

We deduce that a necessary condition for the Einstein relation to hold is that

$$
\int \psi_{p}^{*} \chi_{q} d \mu=\int \psi_{q} \chi_{p} d \mu, \quad \forall p, q=1, \ldots, d .
$$

For example, if $\mu$ is reversible, $\psi^{*}=\psi$, and the above equation is automatically satisfied.

Example 4.2. Tagged particle in interacting Brownian motions with creation-annihilation of particles. This example is basically the continuous version of the model we have studied in section 2. The configuration space of the position of the particles is here given by

$$
\Omega:=\left\{\omega \subset \mathbb{R}^{d}: \operatorname{card}(\omega \cap A)<\infty \text { for all bounded subset } A \text { of } \mathbb{R}^{d}\right\} .
$$

Given $\omega \in \Omega$, a point $x \in \omega$ and $h \in \mathbb{R}^{d}$, we define

$$
\omega^{x, h}:=\left(\omega^{x} \backslash\{x\}\right) \cup\{x+h\}
$$

as the configuration $\omega$ where the point $x$ has been changed to $x+h$. The derivative of a function $f(\omega)$ with respect to a point $x \in \omega$ is defined as

$$
\partial_{x_{p}} f(\omega)=\lim _{\delta \rightarrow 0} \delta^{-1}\left(f\left(\omega^{x, \delta \mathbf{e}_{p}}\right)-f(\omega)\right)
$$

Let $V$ be a finite range stable smooth pair potential, then the formal Hamiltonian is given by

$$
H(\omega)=\sum_{\{x, y\} \subset \omega} V(x-y)
$$

The dynamics is given by the Markov process on $\Omega$ generated by

$$
L=L^{b}+L^{g}
$$


where

$$
L^{b} f(\omega)=\sum_{x \in \omega} \mathrm{e}^{\beta H(\omega)} \partial_{x} \mathrm{e}^{-\beta H} \partial_{x} f(\omega)
$$

and

$$
L^{g} f(\omega)=\sum_{x \in \omega} D_{x}^{-} f(\omega)+z \int \mathrm{e}^{-\beta D_{x}^{+} H(\omega)} D_{x}^{+} f(\omega) d x
$$

with

$$
D_{x}^{-} f(\omega)=f(\omega \backslash\{x\})-f(\omega) \quad D_{x}^{+} f(\omega)=f(\omega \cup\{x\})-f(\omega)
$$

The Gibbs grand canonical measure with temperature $\beta^{-1}$ and activity $z$ is reversible for this dynamics. It follows by the results in [2] that, in a certain range of values for $z$ and $\beta$, there is a spectral gap of this generator. Then one can consider a tagged particle not subject to the Glauber dynamics, and, by applying the method of the present paper, prove the Einstein relation for it. Since this is a quite straightforward extension of what we have done in the previous sections, we omit the details.

Example 4.3. The Einstein relation for a massless tracer particle. Let $\Omega$ be a compact Polish metric space equipped with a group of Borel measurable transformations $\tau_{x}: \Omega \rightarrow \Omega, x \in \mathbb{R}^{d}$. Let $\mu$ be a Borel probability measure on $\Omega$ of which we assume that $U^{x} F(\eta):=F\left(\tau_{x} \eta\right), x \in \mathbb{R}^{d}$ defines a $C_{0}$-group of isometries on any $L^{p}(\mu)$ for $p \in[1,+\infty)$. Let us denote by $C^{m}(\Omega)$ the space consisting of those elements $f \in C(\Omega)$, for which $x \mapsto f\left(\tau_{x} \eta\right)$ possesses $m$ bounded derivatives at $x=0$ that belong to $C(\Omega)$. Let $D f=\left(D_{1} f, \ldots, D_{d} f\right)$ the corresponding differentiation operator, defined as

$$
D_{p} f(\eta):=\lim _{h \rightarrow 0} \frac{f\left(\tau_{h e_{q}} \eta\right)-f(\eta)}{h}, \quad p=1, \ldots, d .
$$

Suppose that $(\omega(t))_{t \geq 0}$ is an $\Omega$-valued continuous trajectory Markov process with transition probability semigroup $\left(P^{t}\right)_{t \geq 0}$. We assume that the dynamics of the process $(\omega(t))_{t \geq 0}$ commutes with the shift transformations, i.e. $P^{t} U^{x}=U^{x} P^{t}$, for all $t \geq 0, x \in \mathbb{R}^{d}$. We also assume that there exists a translation invariant measure $\mu$ on $\Omega$ such that is stationary and ergodic for $P^{t}$. We suppose also that $L$, the $L^{2}(\mu)$-generator of the semigroup, satisfies the spectral gap condition $-(L f, f)_{L^{2}(\mu)} \geq \lambda_{0}\|f\|_{L^{2}(\mu)}^{2}$ for a certain $\lambda_{0}>0$, for all $f \in \mathcal{D}(L) \cap L_{0}^{2}(\mu)$.

Let $\left[a_{p, q}\right]$ a $d \times d$ matrix valued function on $\Omega$ such that there exists $\lambda>0$ and for all $c \in \mathbb{R}^{d}$

$$
\lambda^{-1}|c|^{2} \leq \sum_{p, q} a_{p, q} c_{p} c_{q} \leq \lambda|c|^{2},|c|^{2}=c_{1}^{2}+\ldots+c_{d}^{2} .
$$

We assume that $a \in C^{3}(\Omega)$. Let $\left(x_{t}\right)_{t \geq 0}$ be the diffusion on $\mathbb{R}^{d}$, given by the stochastic differential equation

$$
d x_{t}=\sigma\left(\tau_{x_{t}} \omega(t)\right) d w(t)+\psi\left(\tau_{x_{t}} \omega(t)\right) d t
$$

where $w(\cdot)=\left(w_{1}(\cdot), \ldots, w_{d}(\cdot)\right)$ is a standard $d$-dimensional Brownian motion (independent of $\left.(\omega(t))_{t \geq 0}\right), \sigma=\sqrt{a^{s}}$, where $a^{s}$ is the symmetric part of the matrix $a$, and

$$
\psi_{q}=\sum_{p} D_{p} a_{p, q}
$$


We define the process $\xi_{t}:=\tau_{x_{t}} \omega(t), t \geq 0$. It is standard to show that measure $\mu$ is stationary and ergodic for this process and its $L^{2}(\mu)$ generator equals

$$
\mathcal{L}_{0} f=\frac{1}{2} \sum_{p, q} D_{p}\left(a_{p, q} D_{q} f\right)+L f
$$

for $f \in \mathcal{C}:=C^{2}(\Omega) \cap D(L)$. In addition, $\mathcal{C}$ is a core of the generator. The generator satisfies the spectral gap condition w.r.t. measure $\mu$. Since in this case the position of the tracer is not measurable w.r.t. the natural filtration of $\left(\xi_{t}\right)_{t \geq 0}$ we use relation (1.5) to define the backward mean velocity with $\mathbb{E}_{\mu}$ denoting the path measure corresponding to $\left(\left(x_{t}, \xi_{t}\right)\right)$ with the initial distribution $\delta_{0} \otimes \mu$. After a straightforward calculation it turns out that the backward mean velocity is equal to $\psi_{q}^{*}=\sum_{p} D_{p} a_{q, p}$. So, if $a$ is symmetric, we have $\psi^{*}=\psi$.

Let us fix $\mathbf{l}=\left(\ell_{1}, \ldots, \ell_{d}\right) \in \mathbb{S}^{d-1}, \alpha \in \mathbb{R}$. The argument carried out in section 4 leads us to determine that the perturbation operator is given by

$$
\mathcal{A}_{\alpha} f=\sum_{p, q} a_{p, q}^{s} \ell_{p} D_{q} f
$$

for all $f \in \mathcal{C}$. It is not difficult to check that the assumptions made in section 4 are satisfied.

The position of the tracer in the perturbed system is then given by

$$
d x_{t}^{(\alpha)}=\left(\alpha a^{s}\left(\tau_{x_{t}^{(\alpha)}} \omega\right) \mathbf{l}+\psi\left(\tau_{x_{t}^{(\alpha)}} \omega\right)\right) d t+\sigma\left(\tau_{x_{t}^{(\alpha)}} \omega(t)\right) d w(t)
$$

If $a$ is symmetric and $\mu$ is reversible, since $\psi^{*}=\psi$, the Einstein relation is verified.

Using the above model we can also give an example of a non-reversible system that satisfies the Einstein relation. Let $n \geq 2$ be a fixed integer and $\Omega:=\mathbb{T}_{2}^{n} / \equiv$, where $\mathbb{T}_{2}$ is a two dimensional torus, that we identify with $\mathbb{R}^{2} / 2 \mathbb{Z}^{2}$ and $\equiv$ is the equivalence relation between the $n$-tuples that differ only by the permutation of coordinates. We let $\tau_{x}: \Omega \rightarrow \Omega, x \in \mathbb{R}^{2}$ be given by $\tau_{x}\left(x_{1}, \ldots, x_{n}\right):=\left(x_{1}+x, \ldots, x_{n}+x\right)$ with $\omega=\left(x_{1}, \ldots, x_{n}\right)$ ( + is the addition modulo 2). Here $D F(\omega)=\sum_{i=1}^{n} \nabla_{x_{i}} F(\omega)$ for any periodic, symmetric $C^{1}$-smooth function $F$. The process $\omega(t)$ is defined by the evolution of vortices whose positions are described by $n$ independent two dimensional Brownian motions interacting via a skew-gradient of a smooth potential. More specifically we let $\Phi: \mathbb{T}_{2} \rightarrow \mathbb{R}$ be a certain $C^{2}$-smooth function and let $\nabla^{\perp} \Phi:=\left(\partial_{2} \Phi,-\partial_{1} \Phi\right)$. We let $\omega(t)=\left(x_{1}(t), \ldots, x_{n}(t)\right)$, where

$$
d x_{i}(t)=\sum_{j=1}^{n} \nabla^{\perp} \Phi\left(x_{i}(t)-x_{j}(t)\right) d t+d w_{i}(t), \quad i=1, \ldots, n
$$

and $w_{1}(t), \ldots, w_{n}(t)$ are $n$ independent, standard, two dimensional Brownian motions. The generator of $\omega(t)$ is given by

$$
L F(\omega)=\frac{1}{2} \sum_{i=1}^{n} \partial_{x_{i}}^{2} F(\omega)+\sum_{i, j=1}^{n} \nabla^{\perp} \Phi\left(x_{i}-x_{j}\right) \cdot \nabla_{x_{i}} F(\omega),
$$

for any $F \in C_{2}\left(\mathbb{T}_{2}^{n}\right)$ that is symmetric. It is easy to see that the probability measure $d m_{n}$ induced on $\Omega$ by the $2 n$-dimensional Lebesgue measure is invariant under $(\omega(t))_{t \geq 0}$ and for 
any $F$ having $m_{n}$ zero average we have

$$
-(L F, F)_{L^{2}\left(m_{n}\right)}=1 / 2 \sum_{i=1}^{n} \int_{\Omega}\left(\partial_{x_{i}} F\right)^{2} d m_{n} \geq \gamma\|F\|_{L^{2}\left(m_{n}\right)}^{2},
$$

where the constant $\gamma>0$ is independent of $n$ and $F$. The invariant measure is however non-reversible under the vortex dynamics, for

$$
L^{*} F(\omega)=\frac{1}{2} \sum_{i=1}^{n} \partial_{x_{i}}^{2} F(\omega)-\sum_{i, j=1}^{n} \nabla^{\perp} \Phi\left(x_{i}-x_{j}\right) \cdot \nabla_{x_{i}} F(\omega), \quad F \in C_{2}\left(\mathbb{T}_{2}^{n}\right) .
$$

Let $r(\omega):=-\omega$. Define now $a_{p, q}, p, q=1,2$ to be even functions on $\Omega$, i.e. $a_{p, q}(r(\omega))=$ $a_{p, q}(\omega)$ for all $\omega$ that satisfy (4.26) (then obviously $\psi(r(\omega))=-\psi(\omega)$, cf. (4.28)). Let $R: L^{2} \rightarrow L^{2}$ be given by $R F(\omega):=F(r(\omega))$. It can easily be verified that $R \psi=-\psi, R^{*}=R$ and $R L=L^{*} R$. To check (4.25) note that

$$
\begin{gathered}
\left(\psi_{q}, \chi_{p}\right)_{L^{2}\left(m_{n}\right)}=-\left(R \psi_{q}, \chi_{p}\right)_{L^{2}\left(m_{n}\right)}=\left(R L \chi_{q}, \chi_{p}\right)_{L^{2}\left(m_{n}\right)} \\
=\left(L^{*} R \chi_{q}, \chi_{p}\right)_{L^{2}\left(m_{n}\right)}=\left(\chi_{q}, R L \chi_{p}\right)_{L^{2}\left(m_{n}\right)} \\
=-\left(\chi_{q}, R \psi_{p}\right)_{L^{2}\left(m_{n}\right)}=\left(\chi_{q}, \psi_{p}\right)_{L^{2}\left(m_{n}\right)}, \quad p, q=1,2
\end{gathered}
$$

so (4.25) holds (recall that $\psi^{*}=\psi$ in this case) thus the Einstein relation is satisfied in this case. This example can be easily generalized to an evolution of a system consisting of a random number of vortices on $\mathbb{T}_{2}$ with the initial distribution given by a Poisson measure. Further generalization could be also possible by considering an infinite system of vortices in $\mathbb{R}^{d}$ with the creation and annihilation process analogous to the one presented in Example 4.2

\section{REFERENCES}

[1] O. Benichou, A. M. Cazabat, A Lemarchant, M. Moreau, G. Oshanin Biased Diffusion in a Onedimensional Adsorbed Monolayer. Journ. of Stat. Physics, 97 (1999) 351-371.

[2] L. Bertini, N. Cancrini, F. Cesi, The spectral gap for a Glauber dynamics in a continuous gas, Ann. Inst. H. Poincare (Probabilités et Statistiques) 38 (2002), n. 1, 91-108.

[3] P. Buttà, E. Caglioti, C. Marchioro, On the motion of a charged particle interacting with an infinitely extended system. Comm. Math. Phys. 233 (2003), no. 3, 545-569.

[4] A. De Masi, P.A. Ferrari, S. Goldstein, W.D. Wick, (1989) An invariance principle for reversible Markov precesses. Applications to random walks in random environments, J. Statist. Phys. 55, no.3/4, p 787-855.

[5] A. Einstein, Ann. d. Phys., 17 (1905), 549-560.

[6] A. C. Fannjiang, T. Komorowski, Turbulent diffusion in Markovian flows. Ann. of Appl. Prob. 9 (1999) 591-610.

[7] W. Feller (1966), An introduction to probability theory and its applications. Vol. II, Wiley and Sons, New York.

[8] C. Kipnis, S. R. S. Varadhan, Central Limit Theorem for Additive Functionals of Reversible Markov Processes and Applications to Simple Exclusions. Comm. Math. Phys. 104 (1986) 1-19.

[9] C. Kipnis, C. Landim, Scaling Limits of Interacting Particle Systems, Springer Verlag, Berlin 1999.

[10] Lebowitz, J. L.; Rost, H. The Einstein relation for the displacement of a test particle in a random environment, Stochastic Process. Appl. 54 (1994), no. 2, 183-196.

[11] Liggett, T. M., Interacting Particle Systems. Grunglehren der Mathematischen Wissenschaften, 276, Springer, New York-Berlin (1985).

[12] Liggett, T. M., Stochastic Interacting Systems, Springer, New York-Berlin (1999). 
[13] Loulakis M., Einstein Relation for a tagged particle in simple exclusion processes. Comm. Math. Phys., 229 (2002), 347-367.

[14] Loulakis M., Mobility and Einstein relation for a tagged particle in asymmetric mean zero random walk with simple exclusion, preprint (2003), to appear in Annales de l'Institut H. Poincare, Probabilits et Statistiques.

[15] Olla, S., Homogenization of Diffusion Processes in Random Fields. Manuscript of Centre de Mathématiques Appliquées (1994). Available at http://www.ceremade.dauphine.fr/〜olla/lho.ps

[16] Spohn, H., Large Scale Dynamics of Interacting Particles, Springer, Berlin-Heidelberg 1991.

Instytut Matematyki, PAN

ŚNIADECKICH 8, 00-950 WARSAW, POLAND

Institute of MATHEMATiCs, UMCS

Pl. Mari Curie SkŁodowskiej 1, 20-031 Lublin, Poland

komorow@hektor. umcs.lublin.pl

http://golem.umcs.lublin.pl/ ^komorow

Ceremade, UMR CNRS 7534

Université de Paris Dauphine,

Place du Maréchal De Lattre De Tassigny

75775 Paris Cedex 16 - France.

olla@ceremade.dauphine.fr

http://www . ceremade.dauphine.fr/ olla 